\title{
MicroRNA-361 reduces the viability and migratory ability of pancreatic cancer cells via mediation of the MAPK/JNK pathway
}

\author{
JUAN WANG $^{1}$, ZONGJING XIE ${ }^{2}$, YAN LIU ${ }^{3}$, \\ WEIGUO ZHANG ${ }^{4}$ and TINGTING JI ${ }^{5}$
}

\author{
${ }^{1}$ Department of Gastroenterology, People's Hospital of Leling City, Dezhou, Shandong 253600; \\ ${ }^{2}$ Department of General Surgery, Zhucheng People's Hospital, Weifang, Shandong 262200; ${ }^{3}$ Department of \\ Gastroenterology, Qiqihar Jianhua Hospital, Qiqihar, Heilongjiang 161000; ${ }^{4}$ Second Department of General Surgery, \\ Tianjin Fifth Central Hospital, Tianjin 300450; ${ }^{5}$ Department of Gastroenterology, No. 215 Hospital of \\ Shaanxi Nuclear Industry, Xianyang, Shaanxi 712000, P.R. China
}

Received November 26, 2019; Accepted June 19, 2020

DOI: $10.3892 /$ etm.2021.10799

\begin{abstract}
Previous research has revealed that microRNA-361 (miR-361) functions as a fundamental modulator in non-small-cell lung cancer and esophageal carcinoma. However, its involvement in pancreatic cancer (PC) is yet to be elucidated. Therefore, the present study aimed to examine the mechanism and function of miR-361 during the regulation of PC cell migration and viability. It was demonstrated that miR-361 expression decreased in PC cell lines and tissues, and the overexpression of miR-361 suppressed in vivo PC cell proliferation in mice. Moreover, flow cytometry and MTT assays indicated that the miR-361 mimic decreased the viability and increased the apoptosis of PC cells. Both Transwell migration and wound healing assays identified that miR-361 ameliorated the migratory ability of PC cells. Using dual-luciferase reporter assays, it was found that miR-361 targeted mitogen-activated protein kinase (MAPK)/JNK 3'-untranslated regions, inducing the downregulation of this gene. In PC cells, overexpression of MAPK/JNK diminished the pro-apoptotic effect of the miR-361 mimic, while restoring the migratory activity of PC cells. Collectively, the present results suggested novel molecular mechanisms underlying PC progression and development.
\end{abstract}

Correspondence to: Dr Tingting Ji, Department of Gastroenterology, No. 215 Hospital of Shaanxi Nuclear Industry, 52 Weiyang West Road, Xianyang, Shaanxi 712000, P.R. China E-mail: fs08zr5@163.com

Key words: microRNA-361, pancreatic cancer, mitogen-activated protein kinase/JNK, apoptosis, migration

\section{Introduction}

Pancreatic cancer (PC) results in a relatively low 5-year survival rate (2-9\%) and is one of the most fatal cancer types, ranking as the 7th highest cause of cancer-associated mortality worldwide $(1,2)$. Moreover, there has been a rapid increase $(1.46$ to 7.21 per 100,000$)$ in the occurrence of PC in China over the past several decades $(3,4)$. Although the understanding of $\mathrm{PC}$ has increased, its prognosis remains unsatisfactory, mainly due to the lack of specific symptoms and curative therapies, as well as delayed diagnoses $(5,6)$. Therefore, the discovery of novel PC biomarkers may be a promising tool to facilitate the establishment of more accurate and reliable therapeutic options.

Similar to other types of non-coding RNA molecules ( $<22-25$ nucleotides), microRNAs (miRNAs/miRs) possess post-transcriptional functions that can affect the expression levels of specific genes (7). In non-small-cell lung cancer (NSCLC), a reduction in miR-361 expression has been observed in tumor cells and tissues, and overexpression of miR-361 inhibits the proliferative and migratory abilities of NSCLC cells (8). miR-361 is considered to directly target the Wilms' tumor 1 (WT1) gene in NSCLC. Moreover, WT1 knockdown has a similar influence on miR-361 overexpression in NSCLC cells (8). In esophageal carcinoma tissues, the expression of GLI family zinc finger 1 (Gli1) is notably increased, while that of miR-361 is decreased, corresponding with the TNM stage (9). Additionally, miR-361 reduces Gli1 expression by targeting the 3 --untranslated region (UTR) of the Glil gene (9). With the transfection of small interfering RNA-Gli1 and/or miR-361 mimics, it has been shown that malignant tumor cell development is significantly inhibited (9). Furthermore, miR-361 overexpression and/or Gli1 silencing decreases the cellular production of N-cadherin, Gli1 and Snail, while increasing E-cadherin expression, to suppress the epithelial-mesenchymal transition process and invasive ability of cancer cells; the opposite effects are achieved via Gli1 overexpression (9). However, the effect of miR-361 on PC is yet to be elucidated. 
The present study assessed the role of miR-361 on PC progression. The expression of miR-361 was determined in PC cells and in specimens obtained from patients with PC. Moreover, the effect of miR-361 overexpression on the proliferation of PC cells, as well as impaired PC cell migration, were tested in vitro and in animal models. Therefore, the current study provided evidence that miR-361 was involved in $\mathrm{PC}$ progression in vitro and in vivo.

\section{Materials and methods}

Experimental samples. Patients ( $\mathrm{n}=30 ; 15$ males; 15 females; age range, 40-71 years; median age, 58 years) were selected from histopathologically and clinically diagnosed patients with PC who underwent a primary resection at the No. 215 Hospital of the Shaanxi Nuclear Industry between June 2015 and January 2019. The inclusion criteria for the current study was patients diagnosed with PC. Patients who were treated with radiotherapy or chemotherapy before surgery were excluded. Relevant clinical data were collected, as presented in Table I.

Healthy non-cancerous pancreatic tissue was obtained from volunteers at the No. 215 Hospital of the Shaanxi Nuclear Industry between September 2018 and January 2019 ( $n=10 ; 7$ males; 3 females; age range, 39-65 years; median age, 53 years, healthy group) for use in this research. Clinicopathologic classification and stage of tissues were determined according to the current National Cancer Center Network (NCCN), Union for International Cancer Control TNM classification (10). Patients with PC were divided to three groups, according to the guidelines from the NCCN, as follows: i) Poor prognosis $(n=10)$; ii) medium prognosis $(\mathrm{n}=10)$; and iii) improved prognosis $(\mathrm{n}=10)$ groups. All patients provided written informed consent. The present research was conducted according to the Declaration of Helsinki following ethical approval by the Board of Ethics Review of the No. 215 Hospital of the Shaanxi Nuclear Industry.

Cell culture, transfection and lentiviral infection. DMEM (Gibco; Thermo Fisher Scientific, Inc.) was supplemented with $1 \%$ penicillin/streptomycin (Gibco; Thermo Fisher Scientific, Inc.), $1 \%$ glutamine and 10\% FBS (Gibco; Thermo Fisher Scientific, Inc.), which was then used to cultivate PC cell line PANC-1 [American Type Culture Collection (ATCC)] and AsPC-1 (ATCC) cells. Human pancreatic ductal epithelial cell lines (HPDE6-E6E7 clone 6), immortalized by infection with E6/E7 genes of human papillomavirus 16, were kindly provided by Dr Haoming-Li (Beijing Capital University, Beijing, China) and served as normal pancreatic cells control. Then, $50 \mathrm{nmol} / 1 \mathrm{miR}-361 \mathrm{mimic}$ (5'-UCCCCCAGGUGU GAUUCUGAUUU-3') and a corresponding negative control mimic (NC group) (5'-TTCTCCGAACGTGTCACGT-3') were purchased from GenScript Biotech and used to transfect each cell type using Lipofectamine ${ }^{\circledR}$ RNAiMAX (Thermo Fisher Scientific, Inc.), according to the manufacturer's instructions.

Full-length cDNA for human MAPK/JNK was obtained via PCR using PrimeSTAR Max DNA polymerase (Takara Biotechnology Co., Ltd.). Primer sequences were as follows: Forward, 5'-ATGACTCACGGTCGTAGA-3' and reverse, 5'-GGACCTACGGGTCACTTG-3'. Thermocycling conditions were as follows: Initial denaturation at $95^{\circ} \mathrm{C}$ for $5 \mathrm{~min} ; 35$ cycles of $95^{\circ} \mathrm{C}$ for $10 \mathrm{sec}, 61^{\circ} \mathrm{C}$ for $10 \mathrm{sec}$ and $72^{\circ} \mathrm{C}$ for $15 \mathrm{sec}$. Purified PCR products were subsequently cloned into a pcDNA3 plasmid (GenScript Biotech). The construct was verified via sequencing by Genscript Biotech and designated as 'pcDNA3-MAPK/JNK'. PANC-1 and AsPC-1 cells $\left(1 \times 10^{5}\right.$ cells/well) seeded in a 6 -well plate were transfected with $8 \mu \mathrm{g}$ pcDNA3-MAPK/JNK or pcDNA3-NC using Lipofectamine $^{\circledR} 2000$ transfection reagent (Thermo Fisher Scientific, Inc.). At $36 \mathrm{~h}$ post-transfection, subsequent experiments were performed.

MiR-361 mimic oligonucleotides were analyzed and a scrambled NC sequence served as the NC. The oligonucleotides were phosphorylated, annealed and cloned into the pLVX-puro vector (Clontech Laboratories, Inc), designated as 'pLVX-miR-361' and 'pLVX-NC'. Lentiviral-miR-361 and lentiviral-NC particles were produced via triple transfection of 293 T cells (Invitrogen; Thermo Fisher Scientific, Inc.) using the vectors pLVX-miR-361 and pLVX-NC, along with $0.4 \mu \mathrm{g}$ psPAX2 and pMD2.G (GenScript Biotech). For infection experiments, AsPC-1 cells were incubated with $1 \times 10^{4}$ cells $/ \mathrm{ml}$ lentiviral particles (MOI, 8-10) and polybrene $(5 \mu \mathrm{g} / \mathrm{ml})$ in growth medium. After $6 \mathrm{~h}$, the infection medium was discarded and the cells were used for subsequent experimentation.

MTT assay. Cell viability was assessed via MTT assays. Cells were treated with MTT $(0.5 \mathrm{mg} / \mathrm{ml} ; 20 \mu \mathrm{l}$; Sigma-Aldrich; Merck $\mathrm{KGaA}$ ). The supernatant was removed using a pipette and $150 \mu \mathrm{l}$ DMSO was added to the cells. Then, the cell samples were rotated for $10 \mathrm{~min}$ to allow the incorporation of formazan dye. An Infinite M200 microplate reader (Tecan Group, Ltd.) was used to measure the absorbance of the samples at $490 \mathrm{~nm}$.

Apoptosis assay. To characterize apoptotic proportion, PANC-1 and AsPC-1 cells were collected, washed and resuspended. Following the addition of $5 \mu \mathrm{l}$ Annexin V (cat. no. V13242; Thermo Fisher Scientific, Inc.) and $5 \mu 1$ propidium iodide (PI; cat. no. V13242; Thermo Fisher Scientific, Inc.), cells were incubated at room temperature for $20 \mathrm{~min}$ in the dark, washed with PBS and re-suspended with $300 \mathrm{ml}$ of PBS. Cell apoptosis proportion was analyzed using a BD FACSCalibur ${ }^{\mathrm{TM}}$ flow cytometer (BD Biosciences) equipped with FACS Diva software (version no. 6.0; BD Biosciences) to measure the percentages of early and late apoptotic cells.

Transwell invasion assay. After transfection for $24 \mathrm{~h}, \mathrm{PC}$ cells were trypsinized and washed with D-Hanks solution (Gibco; Thermo Fisher Scientific, Inc.). Wells of 24-well plates were divided into an upper and bottom chamber with Matrigel inserts (pore size, $8 \mu \mathrm{m}$ ). The cell suspension of PC cells $\left(5 \times 10^{5}\right.$ cells) in DMEM was added to the upper chamber. F-12 medium $(400 \mu 1)$ supplemented with FBS (10\%; Thermo Fisher Scientific, Inc.) and hepatocyte growth factor $(20 \mathrm{ng} / \mathrm{ml}$; Thermo Fisher Scientific, Inc.) was added to the bottom compartment. After incubation for $24 \mathrm{~h}$ at $37^{\circ} \mathrm{C}$, cells that migrated from the upper chamber to the lower one were stained with crystal violet. Cell migration was evaluated with a light inverted microscope (Zeiss AG; magnification, $\mathrm{x} 200$ ). 
Table I. Association of miR-361 expression with clinicopathologic features of patients with pancreatic cancer.

\begin{tabular}{|c|c|c|c|}
\hline Characteristic & $\begin{array}{c}\text { Low miR-361, } \\
\text { n }(\%)\end{array}$ & $\begin{array}{l}\text { High miR-361, } \\
\mathrm{n}(\%)\end{array}$ & P-value \\
\hline \multicolumn{4}{|l|}{ Age, years } \\
\hline$\leq 63$ & $8(26.7)$ & $10(33.3)$ & \multirow[t]{2}{*}{0.39} \\
\hline$>63$ & $6(20.0)$ & $6(20.0)$ & \\
\hline \multicolumn{4}{|l|}{ Sex } \\
\hline Male & $5(16.7)$ & $10(33.3)$ & \multirow[t]{2}{*}{0.22} \\
\hline Female & $8(26.7)$ & $7(23.3)$ & \\
\hline \multicolumn{4}{|c|}{ Clinical stage (TNM) } \\
\hline I-II & $2(6.7)$ & $10(33.3)$ & \multirow[t]{2}{*}{0.04} \\
\hline III-IV & $15(50.0)$ & $3(10.0)$ & \\
\hline \multicolumn{4}{|l|}{ Lymph node } \\
\hline Negative & $3(10.0)$ & $4(13.3)$ & \multirow[t]{2}{*}{0.66} \\
\hline Positive & $13(43.3)$ & $10(33.3)$ & \\
\hline \multicolumn{4}{|c|}{ Vascular infiltration } \\
\hline No & $8(26.7)$ & $12(40.0)$ & \multirow[t]{2}{*}{0.56} \\
\hline Yes & $6(20.0)$ & $4(13.3)$ & \\
\hline \multicolumn{4}{|c|}{ Neural infiltration } \\
\hline No & $9(30.0)$ & $12(40.0)$ & \multirow[t]{2}{*}{0.51} \\
\hline Yes & $3(10.0)$ & $6(20.0)$ & \\
\hline \multicolumn{4}{|l|}{ PanIN } \\
\hline No & $5(16.7)$ & $3(10.0)$ & \multirow[t]{2}{*}{0.27} \\
\hline Yes & $16(53.3)$ & $6(20.0)$ & \\
\hline \multicolumn{4}{|c|}{ Grading (AJCC) } \\
\hline $1-2$ & $1(3.3)$ & $13(43.3)$ & \multirow[t]{2}{*}{0.01} \\
\hline 3 & $14(46.7)$ & $2(6.7)$ & \\
\hline \multicolumn{4}{|l|}{ Setting } \\
\hline Metastatic & $5(16.7)$ & $15(50.0)$ & \multirow[t]{2}{*}{0.08} \\
\hline Adjuvant & $6(20.0)$ & $4(13.3)$ & \\
\hline
\end{tabular}

Data were analyzed using a Fisher's exact test. PanIN, pancreatic intraepithelial neoplasia; miR, microRNA; AJCC, American Joint Committee on Cancer.

Wound healing assay. Following $36 \mathrm{~h}$ of transfection, PANC-1 and AsPC-1 cells at a concentration of $1 \times 10^{6}$ cells $/ \mathrm{ml}$ were placed in 24 -well plates and grown to $80 \%$ confluence at $37^{\circ} \mathrm{C}$. Using a sterilized pipette tip, a scratch wound was made. After rinsing with PBS twice, the floating cells were removed. The original medium (with 10\% FBS) was replaced with fresh medium (with $0 \% \mathrm{FBS}$ ) and cells were incubated for 0 (control) or $36 \mathrm{~h}$ at $37^{\circ} \mathrm{C}$. An inverted microscope (Nikon Corporation, magnification, x40) and NIS-Elements (version no. 11.0; Nikon Corporation) was used to obtain and analyze the images of the cells.

Western blot (WB) analysis. Total protein was extracted from cells using cold radioimmunoprecipitation assay buffer (Thermo Fisher Scientific, Inc.). Total protein was quantified using a BCA assay kit (Thermo Fisher Scientific, Inc.) and $50 \mu \mathrm{g}$ protein/lane was separated via $12 \%$ SDS-PAGE. The resultant protein bands were transferred onto PVDF membranes and the membranes were blocked with 5\% non-fat milk in Tris-buffered saline with Tween ${ }^{\circledR} 20$ (Thermo Fisher Scientific, Inc.) for $2 \mathrm{~h}$ at room temperature. After blocking the membranes, they were incubated with primary and secondary antibodies for $2 \mathrm{~h}$ at room temperature. JNK (1:1,000; cat. no. ab112501; Abcam), phosphorylated (p-)JNK (1:500; cat. no. ab4821; Abcam), $\beta$-actin (1:5,000; cat. no. ab8227; Abcam), horseradish peroxidase-conjugated goat anti-rabbit secondary (1:5,000; cat. no. ab6721; Abcam) antibodies were purchased from Abcam. Protein bands were visualized using an ECL western blotting kit (Thermo Fisher Scientific, Inc.) and protein expression was quantified using Image-Pro Plus software (version no. 6.0; Media Cybernetics, Inc.).

RNA extraction and reverse transcription-quantitative PCR (RT-qPCR). TRIzol ${ }^{\circledR}$ reagent (Invitrogen; Thermo Fisher Scientific, Inc.) was used to obtain total RNA from the transfected cells or tissues, according to the manufacturer's protocol. Total RNA $(1 \mu \mathrm{g})$ was reverse transcribed into cDNA using the miScript Reverse Transcription kit (Qiagen, Inc.) for incubation at $37^{\circ} \mathrm{C}$ for $30 \mathrm{~min}$ and inactivated at $95^{\circ} \mathrm{C}$ for $5 \mathrm{~min}$, according to the manufacturer's protocol. The following thermocycling conditions were used 

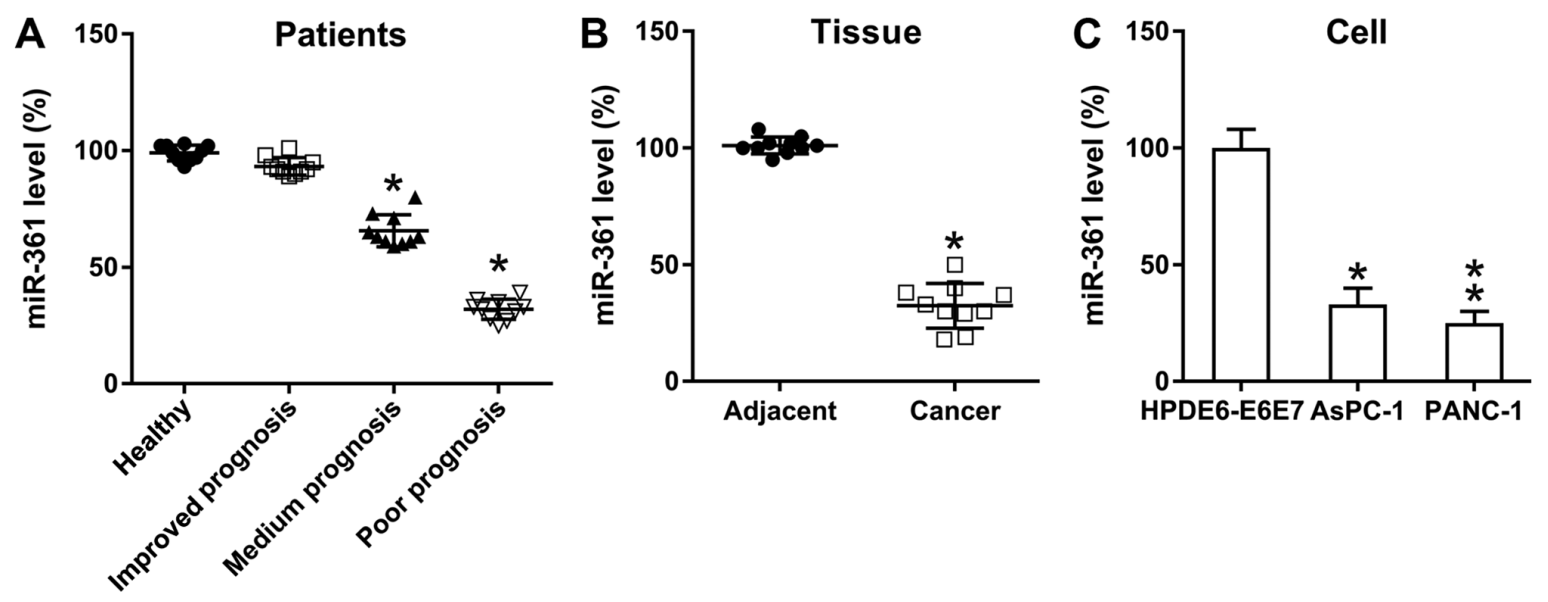

Figure 1. miR-361 expression in PC cells and patient PC tissues. (A) Expression of miR-361 in control subjects and pancreatic cancer cells in the poor, medium and improved prognosis groups (each $n=10$ ) was analyzed using reverse transcription-quantitative PCR. Data were analyzed using one-way ANOVA. (B) Expression of miR-361 from surrounding non-cancerous pancreatic tissues in volunteers compared with PC tissues. Data were analyzed using Student's t-test. (C) Expression of miR-361 in normal pancreatic cells and PC cells. Data were analyzed using one-way ANOVA followed by Dunnett's test. Data are presented as the mean $\pm \mathrm{SD}$. ${ }^{*} \mathrm{P}<0.05$ and ${ }^{* *} \mathrm{P}<0.01$ vs. the healthy/Adjacent/HPDE6-E6E7 group. miR, microRNA.

for the qPCR: Initial denaturation at $95^{\circ} \mathrm{C}$ for $5 \mathrm{~min}$; 40 cycles of $95^{\circ} \mathrm{C}$ for $10 \mathrm{sec}, 60^{\circ} \mathrm{C}$ for $30 \mathrm{sec}$ and $72^{\circ} \mathrm{C}$ for $15 \mathrm{sec}$. The Roche Light-Cycler 480 RT PCR System (Roche; Thermo Fisher Scientific, Inc.) and Fast SYBR ${ }^{\mathrm{TM}}$ Green Master Mix (cat. no. 4385617; Thermo Fisher Scientific, Inc.) were used to determine miR-361 expression. GAPDH was set as the internal control. SYBR Green PCR Master Mix was used for RT-qPCR. The number of targets was measured with respect to the calibrator (average value of the control groups) using the $2^{-\Delta \Delta \mathrm{Cq}}$ method (11). The primers used were as follows: miR-361 forward, 5'-TTGCATAGTCACAAAAGTGAT-3' and reverse, 5'-CAGTGCGTGTCGTGGAGT-3'; U6 forward, 5'-CTCGCT TCGGCAGCACATATACT-3' and reverse, 5'-ACGCTTCAC GAATTTGCGTGTC-3'; JNK forward, 5'-TTTACTTAGGGG TCATAGGTGAG-3' and reverse, 5'-AGACTCCCGCTCTCC AACAAG-3'; and GAPDH forward, 5'-GGAGCGAGATCC CTCCAAAAT-3' and reverse, 5'-GGCTGTTGTCATACT TCTCATGG-3'.

Target Scan prediction. The prediction algorithm TargetScan was used to identify miR-361 targets. TargetScan Database predictions (version no. 7.2; http://www.targetscan.org) are ranked according to the predicted targeting effectiveness as measured using the cumulative weighted context + values of the sites (12). Additionally, conserved targeting probability can be selected to rank the predictions (13).

Dual-luciferase reporter assay (DLRA). The 3'-UTR luciferase reporter assay was performed to determine the target gene for miR-361, wherein the mutant and wild-type (WT) 3'-UTR of MAPK/JNK were inserted in psi-CHECK2 (Genscript). PANC-1 cells were co-transfected with $10 \mu \mathrm{g}$ miR-361 mimic (or NC mimic) and $4 \mu \mathrm{g}$ WT-MAPK/JNK-3'UTR plasmid (or MU-MAPK/JNK-3'UTR plasmid) using Lipofectamine ${ }^{\circledR}$ 2000. Following incubation for $48 \mathrm{~h}$, reporter luminescence was normalized using the Renilla luciferase sequence and calibration luminescence was measured using the firefly luciferase sequence using a Dual-Luciferase Reporter Assay system (Promega Corporation).
Animal experiments. The experimental protocol involving animals was approved by the Animal Ethics Committee of the No. 215 Hospital of Shaanxi Nuclear Industry. Humane endpoints were set as follows: i) Tumor burden (combined burden if $>1$ mass is present) is $>15 \%$ body weight; ii) mean tumor diameter $\geq 20 \mathrm{~mm}$; and iii) tumor ulceration, infection or necrosis. No mice died during the experiments. The duration of the experiment was 28 days.

Nude BALB/c-nu mice ( $n=16$; age, 5 weeks; female; weight, 20-30 g) were purchased from Beijing Vital River Laboratory Animal Technology Co., Ltd. The mice were housed in groups of four in the animal facility for the entire experimental period. All mice were under constant temperature $\left(21^{\circ} \mathrm{C}\right)$ and humidity (45-55\%) with 12-h light/dark cycles, with lights on at 7:00 am. Standard food and filtered water were available ad libitum. AsPC-1 cells $\left(1 \times 10^{6}\right)$ were infected with lentiviral-miR-361 or lentiviral-NC viral particles to generate AsPC-1 cells with constitutive expression of miR-361 (AsPC-1-miR-361) and control cells (AsPC-1-NC), respectively. All mice received a subcutaneous injection (50 $\mu \mathrm{l})$ containing transfected cells in the right armpit during administration of inhalant anesthetics isoflurane (2\%), which was used to induce general anesthesia, after adapting to the environment for 3 days. The tumor size was measured every 3 days using a vernier caliper. The animals were sacrificed by cervical dislocation on day 28 after injection. Death was confirmed by the lack of breathing and corneal reflex. Tumor weights are presented in $\mathrm{g}$ and the formula $\left(\mathrm{LxW}^{2}\right) / 2$ was used to measure tumor size. The maximum tumor diameter observed in mice was $1.05 \mathrm{~cm}$.

Statistical analysis. Experiments were performed in triplicate. Data were analyzed by SPSS Statistics for Windows (version 18.0; SPSS, Inc.). Data are presented as the mean \pm SD. One-way ANOVA followed by a Tukey's or Dunnett's post-hoc test and the two-tailed Student's t-test were used to compare the results of multiple group and two experimental groups, respectively. $\mathrm{P}<0.05$ was considered to indicate a statistically significant difference. 

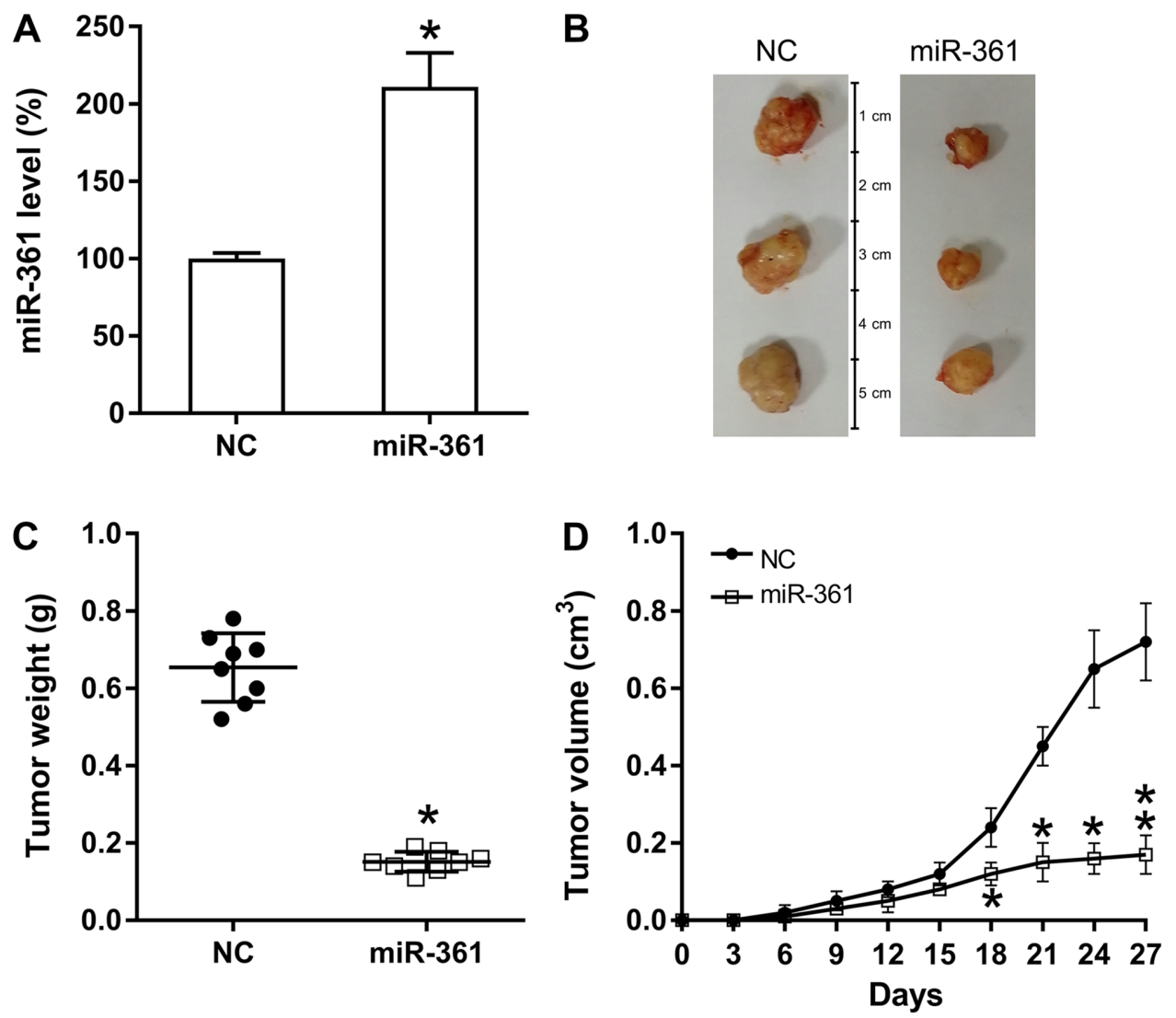

Figure 2. Xenograft pancreatic cancer formation is suppressed by miR-361 in mice. (A) Pancreatic miR-361 expression measured in each group using reverse transcription-quantitative PCR. Data were analyzed using an unpaired t-test. (B) AsPC-1 cells transfected with adenovirus-miR-361 or NC cells were administered to mice subcutaneously ( $\mathrm{n}=8$ in each group). At day 27 post-inoculation, the animals were sacrificed and the tumors were weighed. (C) Tumors from each group were weighed following excision. Data were analyzed using an unpaired t-test. (D) Tumor development curve 27 days post-inoculation. Data were analyzed using mixed ANOVA and a Bonferroni/Sidak post hoc test. Data are presented as the mean $\pm \mathrm{SD}$. " $\mathrm{P}<0.05$ and ${ }^{* *} \mathrm{P}<0.01$ vs. NC group. NC, negative control; miR, microRNA.

\section{Results}

miR-361 expression is decreased in PC specimens and cells. To determine the role of miR-361 on PC development, miR-361 expression in PC tissues was examined using RT-qPCR. In accordance with the guidelines from the NCCN, three test subject groups were established as follows: Poor, medium and improved prognosis groups. Compared with the control group, the medium and poor prognosis groups demonstrated a significantly reduced expression of miR-361 in pancreatic cancer, while the improved prognosis group showed no significant difference (Fig. 1A). Moreover, compared with samples from the adjacent healthy pancreas, $\mathrm{PC}$ specimens presented a significant reduction in miR-361 expression (Fig. 1B), while PANC-1 and AsPC-1 cells also exhibited downregulated miR-361 expression compared with normal pancreatic cells (Fig. 1C). Thus, miR-361 expression was found to be reduced in $\mathrm{PC}$, suggesting a potential role for miR-361 during PC cell proliferation in vivo.

miR-361 suppresses $P C$ cell proliferation in vivo. To assess the influence of miR-361 on the in vivo proliferation of xenograft PC tumor cells, nude BALB/c mice were subcutaneously injected with AsPC-1-miR-361 (stably expressing miR-361) or AsPC-1-NC cells, and observed every 3 days for tumor development and overexpression of miR-361. Pancreatic miR-361 expression was examined in different groups and miR-361 upregulation was identified in the miR-361 group (Fig. 2A). Mice were sacrificed at 28 days following injection and their pancreatic tissues were removed and weighed (Fig. 2B). The results demonstrated that based on the average weight and volume of the tumors, miR-361-generated tumors developed much slower compared with those from mice in the NC group and the average neoplasm volume and weight was significantly lower compared with neoplasms in mice from the $\mathrm{NC}$ groups at day 27 post-injection (Fig. 2C and D).

miR-361 inhibits the viability and triggers apoptosis of PC cells. The influence of miR-361 on PC cell proliferation was analyzed. miR-361 mimic transfection significantly increased the expression of miR-361 in PANC-1 and AsPC-1 cells (Fig. 3A and B). Then, based on the MTT assay results, it was found that ectopic miR-361 overexpression caused a significant decrease in the rate of proliferation of PANC-1 and AsPC-1 cells (Fig. 3C and D).

It was also hypothesized that miR-361 may stimulate apoptosis in these two cell lines. Therefore, both PI flow cytometry and Annexin V-FITC staining were performed to detect the number of apoptotic cells in each group. The results identified a significant increase in the apoptotic rate in PC cells that were transfected with the miR-361 mimic compared with the NC group (Fig. 3E and F). 

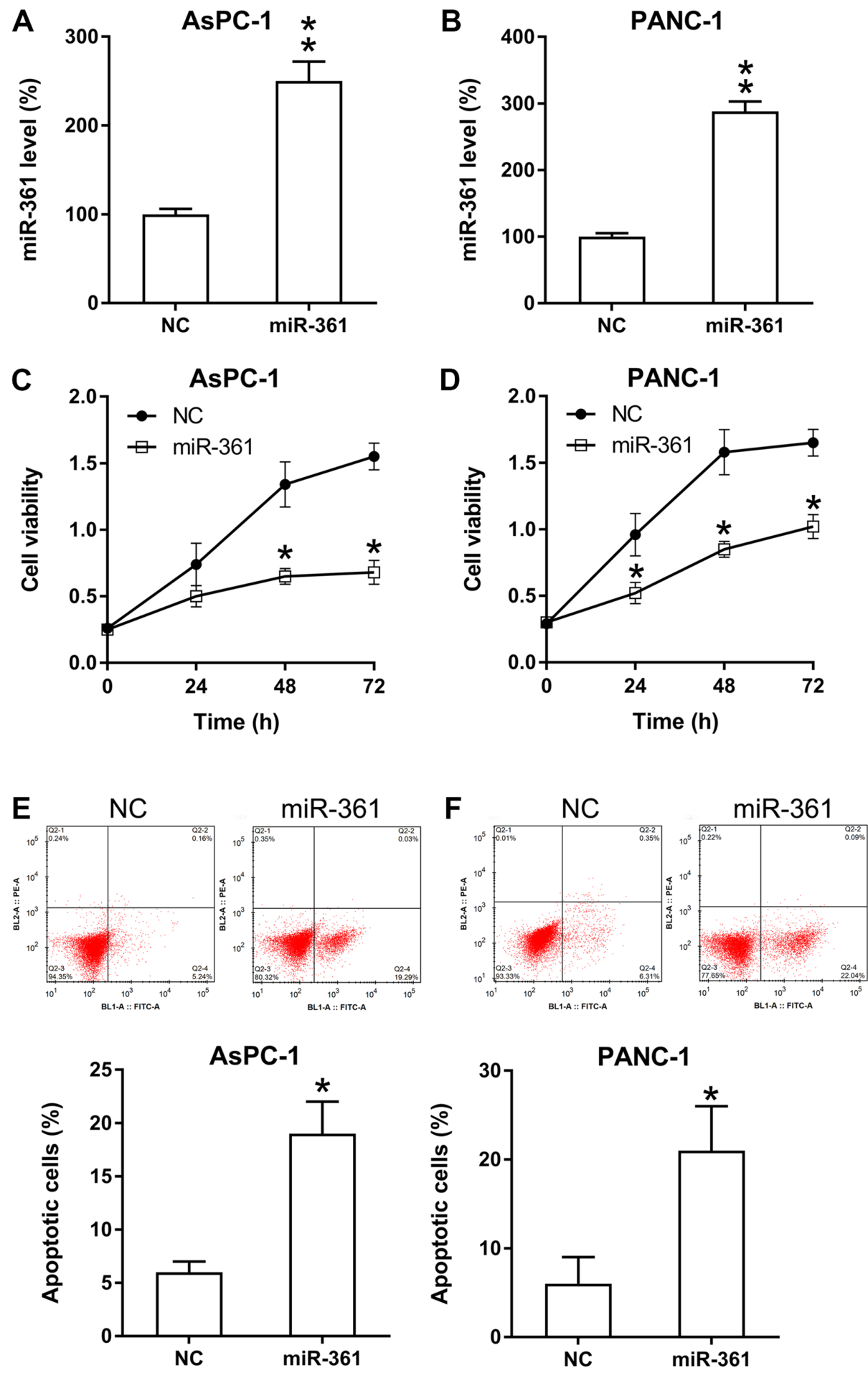

Figure 3. Influence of miR-361 expression on the apoptotic and proliferative abilities of PANC-1 and AsPC-1 cells. Expression of miR-361 in (A) AsPC-1 and (B) PANC-1 cells following transfection with NC mimic or miR-361 mimic, as determined by reverse transcription-quantitative PCR. Data were analyzed using unpaired t-test. Rate of proliferation in (C) AsPC-1 and (D) PANC-1 cells determined at 0,24,48 and $72 \mathrm{~h}$ after transfection via the MTT assay. Annexin V-FITC and PI flow cytometry were performed to assess the number of apoptotic (E) AsPC-1 and (F) PANC-1 cells. Upper right quadrant indicates the early apoptotic cells. Data were analyzed using unpaired t-test. Data are presented as the mean $\pm \mathrm{SD}$. ${ }^{\mathrm{P}}<0.05$ and ${ }^{* *} \mathrm{P}<0.01$ vs. NC group. NC, negative control; miR, microRNA; PI, propidium iodide.

miR-361 inhibits the migratory ability of PC cells. To assess whether the migratory process of PC cells is affected by miR-361, wound-healing and Transwell migration assays were conducted to measure the migration of two PC cell lines following transfection. miR-361 overexpression demonstrated a significant inhibitory influence on the migratory capabilities of both PANC-1 and AsPC-1 cells at $36 \mathrm{~h}$, as determined using wound-healing assays (Fig. 4A and B). Additionally, the Transwell migration assay identified that the miR-361 mimic induced a significant decrease in PC cell migration (Fig. 4C and D). These findings suggested that miR-361 suppressed the migration of PC cells. 
A
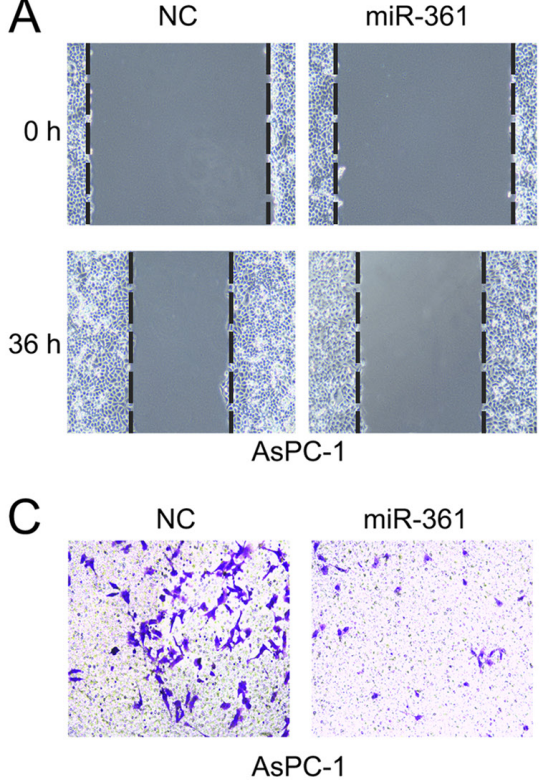

B

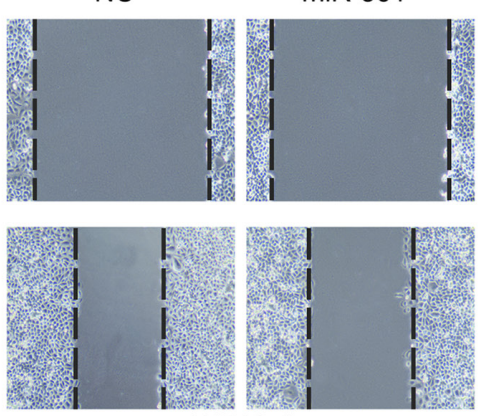

PANC-1

D

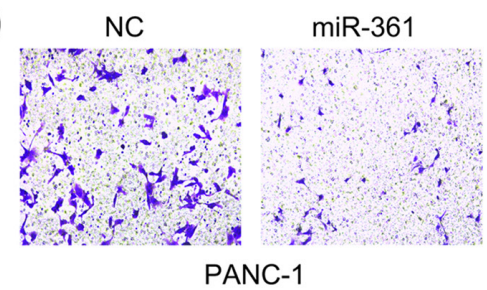

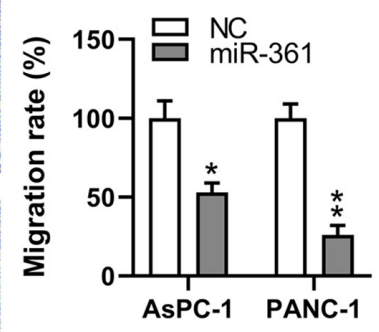

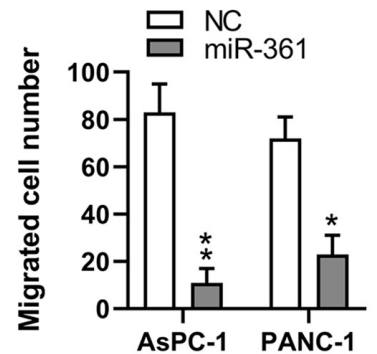

Figure 4. Migratory process of PC cells was inhibited by miR-361. Migration of transfected (A) PANC-1 and (B) AsPC-1 cells was measured via wound-healing assay. Magnification, x40. Migration of (C) PANC-1 and (D) AsPC-1 cells was also measured using a Transwell migration assay following transfection with miR-361 mimic or NC mimic. Data were analyzed using unpaired t-test. Magnification, $\mathrm{x} 200$. Data are presented as the mean $\pm \mathrm{SD}$. ${ }^{*} \mathrm{P}<0.05$ and ${ }^{* *} \mathrm{P}<0.01$ vs. NC group. NC, negative control; miR, microRNA.

A

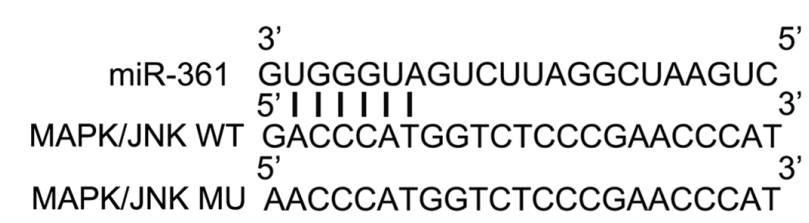

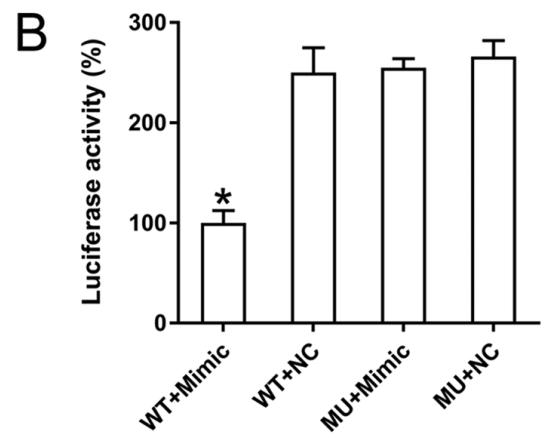
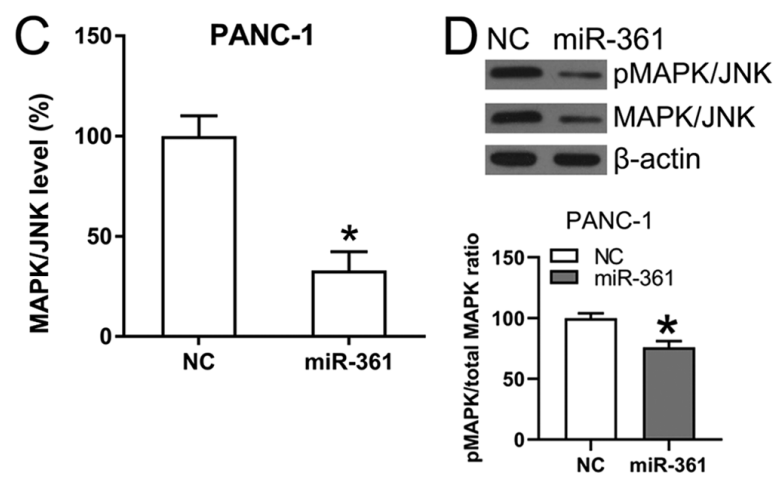

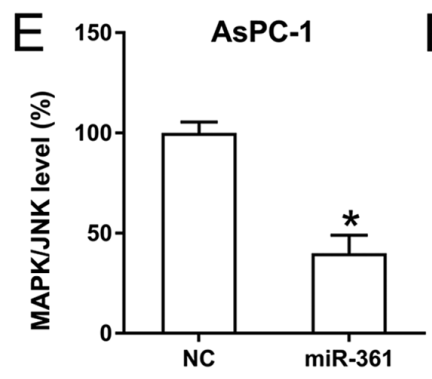

F NC miR-361

-

$=$ MAPK/JNK

S-actin

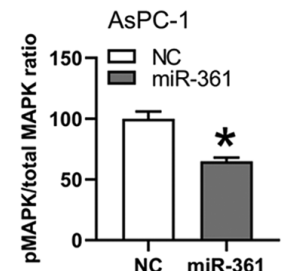

Figure 5. MAPK/JNK is targeted by miR-361. (A) Conserved miR-361 binding motif on the 3'-UTRs of MAPK. (B) Following the transfection of the miR-361 mimic, luciferase activity was assessed using the luciferase reporter construct, which contains either the MU or WT copy of the human MAPK/JNK 3'-UTRs. The luciferase activity was standardized to the Renilla luciferase activity. Data were analyzed using one-way ANOVA. (C) RT-qPCR and (D) WB analyses were performed to assess the expression and phosphorylation of MAPK/JNK following transfection of PANC-1 cells using the NC mimic or the miR-361 mimic. (E) RT-qPCR and (F) WB analyses were performed to assess the expression and phosphorylation of MAPK/JNK following transfection of AsPC-1 cells using the NC mimic or the miR-361 mimic. Data were analyzed using unpaired t-test. Data are presented as the mean $\pm \mathrm{SD}$. "P<0.05 vs. NC groups. $\mathrm{NC}$, negative control; miR, microRNA; WT, wild-type; MU, mutant; UTR, untranslated region; MAPK, mitogen-activated protein kinase; p, phosphorylated; RT-qPCR, reverse transcription-quantitative PCR; WB, western blot.

miR-361 targets the 3'-UTR of MAPK/JNK. MAPK/JNK has been reported to be involved in apoptosis in various cancer cells (14-16). Therefore, it was hypothesized that
MAPK/JNK may be involved in miR-361-mediated apoptosis and metastasis of PC cells. Bioinformatic predictions indicated that miR-361 possesses the ability to target the 

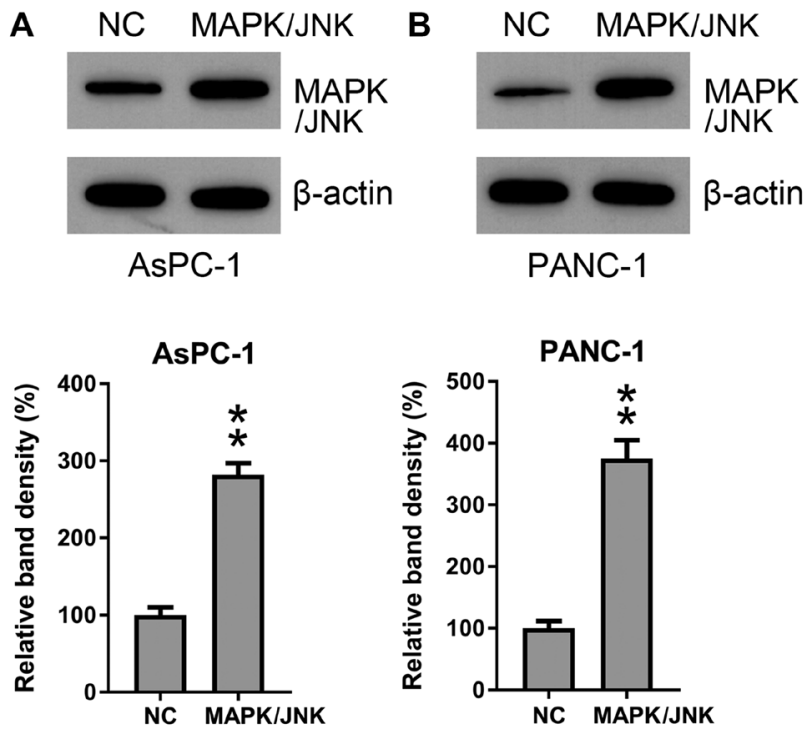

Figure 6. Overexpression of MAPK/JNK in PC cells. Cells were transfected using a pcDNA3-MAPK/JNK or pcDNA3, without any other treatment. MAPK/JNK expression was measured using western blotting in (A) PANC-1 and (B) AsPC-1 cells. Data were analyzed using unpaired t-test. Data are presented as the mean $\pm \mathrm{SD}$. ${ }^{* *} \mathrm{P}<0.05$ vs. $\mathrm{NC}$ group. $\mathrm{NC}$, negative control; MAPK, mitogen-activated protein kinase.

3'-UTR in MAPK/JNK (Fig. 5A). Furthermore, DLRA found a direct interaction between the WT-MAPK/JNK 3'-UTR and miR-361 (Fig. 5B) and that luciferase function was inhibited following transfection with the miR-361 mimic, which bound to the WT-MAPK/JNK 3'-UTR with $<50 \%$ affinity compared with the control groups.

The effect of ectopic overexpression of miR-361 on MAPK/JNK expression was also analyzed in AsPC-1 and PANC-1 cells using RT-qPCR and WB analysis. MAPK/JNK mRNA and protein expression levels were significantly downregulated as a result of the transfection with the miR-361 mimic (Fig. 5C-F). Thus, it was found that MAPK/JNK expression decreased following miR-361 overexpression.

MAPK/JNK restores PC cell viability and metastasis attenuated by miR-361. To determine whether MAPK/JNK participated in the effects induced by miR-361 on PC cell viability and metastasis, MAPK/JNK was overexpressed in the AsPC-1 and PANC-1 cells. WB analysis results demonstrated the successful overexpression of MAPK/JNK (Fig. 6A and B). MAPK/JNK was overexpressed in AsPC-1 and PANC-1 cells that were also co-transfected with the miR-361 mimic and WB analyses were conducted to confirm MAPK/JNK upregulation in cells (Fig. 7A and B).

Flow cytometry was used to detect the quantity of apoptotic cells. While MAPK/JNK was overexpressed and pMAPK/JNK expression was significantly increased (Fig. 7A and B), the apoptotic process induced by miR-361 in AsPC-1 and PANC-1 cells was significantly impeded in cells transfected with MAPK/JNK overexpression vector + miR-361 mimic (Fig. 7C and D).

To evaluate the role of MAPK/JNK in the process of PC cell metastasis, Transwell migration and would healing assays were performed on two PC cell lines following their transfection with different constructs. Cells overexpressing
MAPK/JNK + miR-361 demonstrated restored migratory abilities compared with the NC groups (Fig. 7E-H).

\section{Discussion}

$\mathrm{PC}$ is a malignant condition that severely affects the health of patients (16). Only $1 / 5$ patients with PC are viable for surgical therapy, which is currently the sole reliable curative therapy for PC (16). Therefore, identifying effective methods of PC inhibition are of great significance. However, the limitation of the present study was the lack of animal experiments, which will be performed in future studies.

Previous studies have reported that dysregulated miRNA expression may cause the development and progression of PC carcinoma (17-20). Thus, the discovery of tumor-associated miRNAs, assessment of their therapeutic effects and identification of their target mRNAs will be important for finding novel therapeutic targets (20). It has been revealed that miRNAs perform vital functions with regard to various biological activities involved in PC progression. For instance, miR-195 expression is decreased in PC cell lines and tissues (21). Furthermore, increased expression of miR-195 suppresses PC invasion and proliferation by modulating the Wnt/fatty acid synthase signal pathway (21) The expression of miR-181d is increased not only in PC cells, but also in human PC (22). It has also been shown that downregulation of miR-181d induced via transfection with specific lentiviral constructs inhibits the processes of fluorouracil resistance, migration and proliferation in PC cells, and prevents the tumor explant from growing in animal models (23). Additionally, miR-181d can directly target sodium/potassium transporting ATPase interacting 2 (NKAIN2) in PC, while NKAIN2 knockdown mediated by small interfering RNA could reverse the suppression of miR-181d downregulation during PC development (22). PC utilizes autophagy as a means of survival to generate fundamental glucose necessary for glycolysis metabolism and it has been revealed that miR-7 inhibits autophagy via activating the serine/threonine kinase 11/AMP-activated protein kinase/mTOR signaling pathway in stressful tumor microenvironments (23). miR-7 also directly targets specific stages of cellular autophagy induction and vesicle elongation to decrease glucose provision in cells involved in glycolysis metabolism (24). Moreover, miR-7 suppresses PC cell metastasis and proliferation in vitro and in animal models (24). The present results demonstrated that the expression of miR-361 was decreased in PC cells and tissues and that miR-361 overexpression significantly inhibited the viability and migration of PC cells. In addition, MAPK/JNK was found to be the direct target of miR-361. Thus, it was suggested that miR-361 may function as a PC inhibitor and could be a promising target in PC treatment.

miR-361 is located in Xq21.2, an intron located between exon 10 and exon 9 in the choroideremia gene (2). A decrease in miR-361 is observed in various human malignancies, including liver carcinoma (25), gastric tumor (26), colorectal malignancy (26), prostate carcinoma (27), NSCLC (8), esophageal tumors (9) and cutaneous squamous cell carcinoma (24). Its targets include, but are not limited to, SH2B adaptor protein 1 in NSCLC and colorectal cancer $(28,29)$, ERK1/1 in pancreatic ductal adenocarcinoma (30), hedgehog 
A
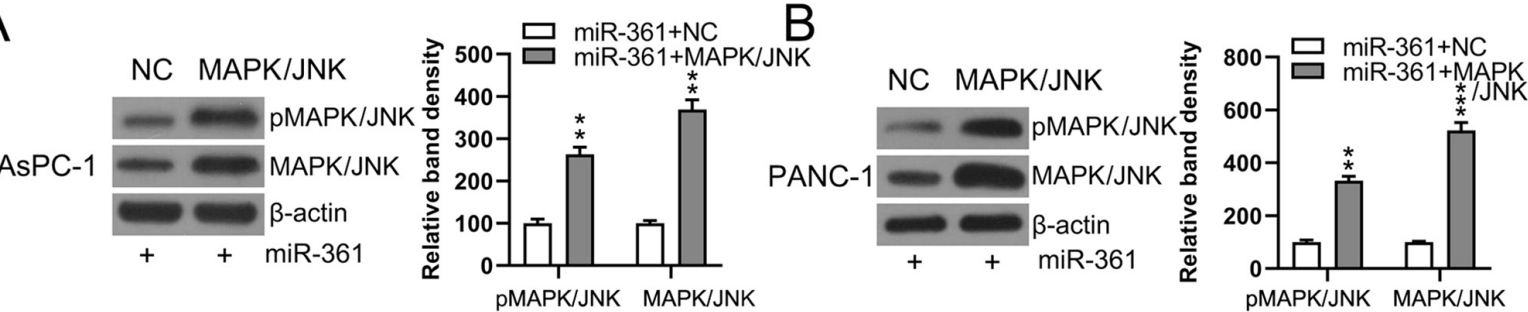

C
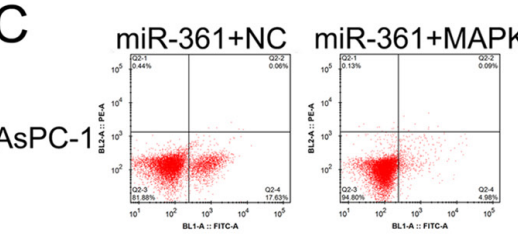

NN D
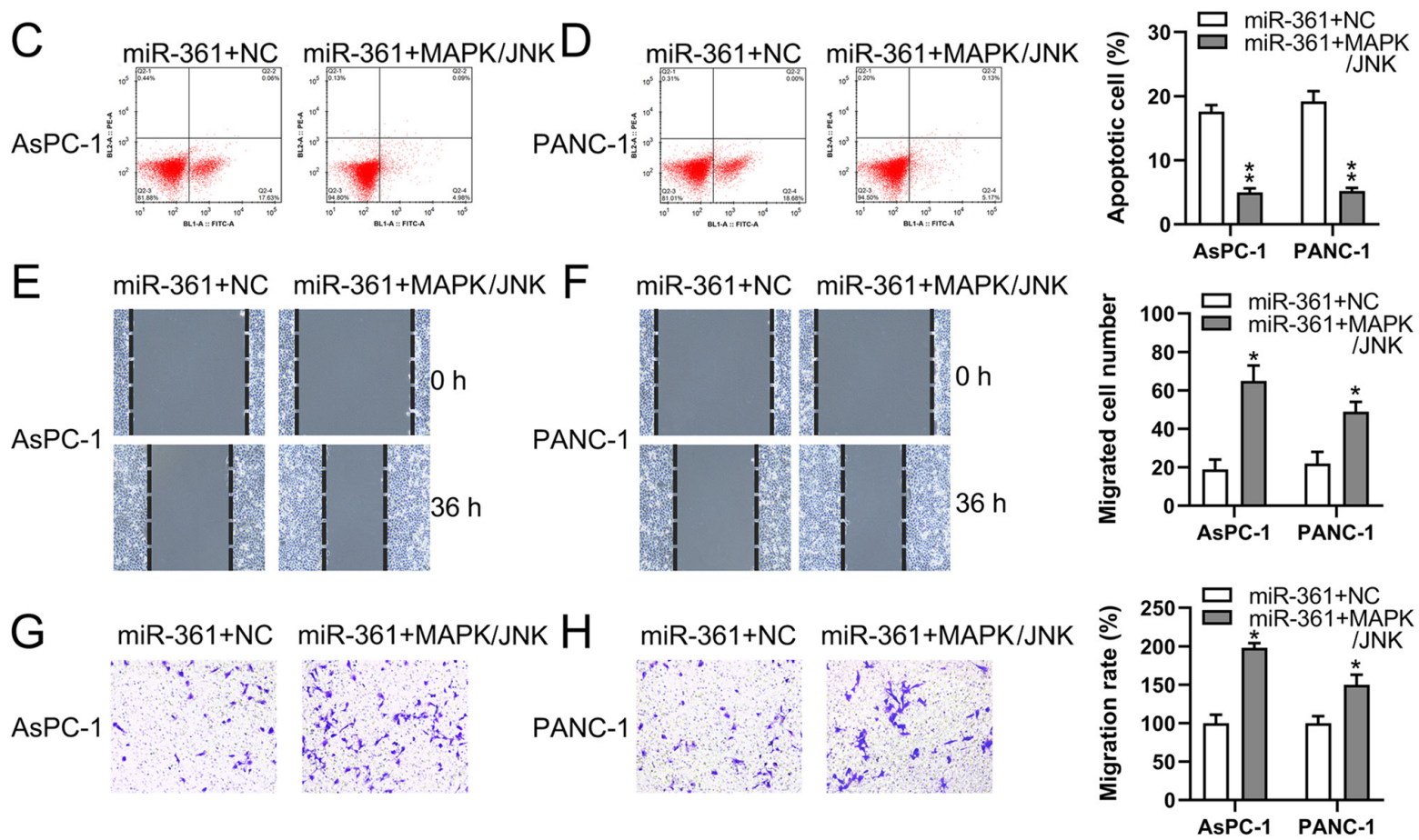

Figure 7.Expression of MAPK/JNK increases the viability and metastasis of PC cells. Cells were co-transfected using a miR-361 mimic and pcDNA3-MAPK/NC. MAPK/JNK expression and phosphorylation was measured using western blotting in co-transfected (A) AsPC-1 and (B) PANC-1 cells. Annexin V-FITC and PI flow cytometry determined the number of apoptotic (C) AsPC-1 and (D) PANC-1 cells transfected with different constructs. Wound healing assays were performed to measure the migratory ability of (E) AsPC-1 and (F) PANC-1 cells at 0 and 36 h post-transfection. Magnification, $\mathrm{x} 40$. (G) AsPC-1 and $(\mathrm{H})$ PANC-1 cell migration was determined using Transwell migration assay at $36 \mathrm{~h}$ post-transfection. Magnification, $\mathrm{x} 200$. Data were analyzed using unpaired t-test. Data are presented as the mean $\pm \mathrm{SD} .{ }^{*} \mathrm{P}<0.05,{ }^{* *} \mathrm{P}<0.01$ and ${ }^{* * *} \mathrm{P}<0.01$ vs. miR-361 + NC group. NC, negative control; miR, microRNA; MAPK, mitogen-activated protein kinase; $\mathrm{p}$, phosphorylated; PI, propidium iodide.

in retinoblastoma (31), granzyme B in cutaneous leishmaniasis (32) and $\beta$-secretase 1 in Alzheimer's disease (33). It has been reported that miR-361 may regulate $\mathrm{C}-\mathrm{X}-\mathrm{C}$ motif chemokine receptor 6 to inhibit liver tumor cell proliferation, while another study revealed that miR-361 regulated STAT6 expression by functioning as a tumor inhibition gene in prostate tumors. Moreover, Ma et al (26) found that miR-361 could decrease the recurrence rate of gastric cancer via interacting with the CD80 gene 3'-UTR. Furthermore, results showed that miR-361 could specifically inhibit staphylococcal nuclease domain containing-1 gene expression and growth of colorectal tumor bodies. Collectively, these results indicated that miR-361 serves a role in suppressing the growth of multiple tumor types.

The present study also identified an inhibitory role of miR-361 in PC. MTT assay results demonstrated that after miR-361 overexpression using transfection with mimics, the viability of AsPC-1 and PANC-1 cells significantly decreased. Flow cytometry also identified that miR-361 could induce PC cell apoptosis. Similar effects were also found with regard to the migratory abilities of PC cells, which were inhibited by miR-361, suggesting that miR-361 served an inhibitory role in PC. However, a previous report revealed that miR-361 overexpression promoted PC cell migration and invasion in vitro (PANC-1, SW1990, BxPC-3 and CFPAC-1 cells) (30), while conversely, the present study identified an inhibitory effect of miR-361 on PC development in vivo. In addition, miR-361 overexpression was found to reduce PC cell proliferation and metastasis. Therefore, it was hypothesized that the discrepancies between these two studies may be attributed to the differences in cell lines and patient cohorts used.

The generic MAPK/JNK signaling pathway is involved in four different cascades, named according to their MAPK/JNK tier constituents: ERK1/2, ERK5, p38 MAPK and c-Jun aminoterminal kinases (34). Furthermore, ERK and MAPK/JNK pathways are associated with cell apoptosis, senescence, migration, differentiation and proliferation (34). It has been reported that the natural compound Oblongifolin $\mathrm{C}$ effectively suppresses PC cell proliferation by inducing apoptosis and $G_{0} / G_{1}$ arrest via amelioration of the Src proto-oncogene/MAPK/ERK signaling pathways (35). 
Sulforaphane is an active component found in cruciferous vegetables and can repress cell colony formation and proliferation, as well as induce apoptosis via caspase-3 activation in PC cells (36). Previous studies have also shown that sulforaphane suppresses AKT and MAPK/JNK phosphorylation and stimulates forkhead transcription factors, causing apoptosis and cell cycle arrest (36). The proto-oncogene Kirsten rat sarcoma viral oncogene (KRAS) serves an important role in PC formation and development and the ERK/MAPK/JNK signaling pathway participates in modulating KRAS expression in PC (37). Thus, these studies support the conclusion that MAPK/JNK exerts a protective role in PC growth and metastasis. In the current study, MAPK/JNK expression was repressed via transfection with the miR-361 mimic. Overexpression of MAPK/JNK restored the proliferative and migratory capabilities in PC cells suppressed by miR-361, suggesting that MAPK/JNK is involved in mediating the activity of miR-361 during PC development.

In conclusion, the present results indicated that miR-361 may possess tumor-suppressing effects that result in impaired major malignant capabilities in PC cell lines and in PC cell proliferation in vivo. Furthermore, the results suggested that decreased miR-361 expression in PC tumors and cells was associated with cancer cell proliferation impairment. Thus, miR-361 may be considered as a prognostic and diagnostic biomarker for PC.

\section{Acknowledgements}

Not applicable.

\section{Funding}

No funding was received.

\section{Availability of data and materials}

The datasets used and/or analyzed during the current study are available from the corresponding author on reasonable request.

\section{Authors' contributions}

JW conceived the current study and designed the experiments. ZX, YL and WZ contributed to the data collection, performed data analysis and interpreted the results. JW wrote the manuscript. TJ contributed to the data collection and analysis during the revision of the article. JW and YL confirm the authenticity of all the raw data. All authors read and approved the final manuscript.

\section{Ethics approval and consent to participate}

The current study was approved by the Board of Ethics Review of the No. 215 Hospital of the Shaanxi Nuclear Industry (Xianyang, China). All patients provided written informed consent prior to enrollment in the current study. The experimental protocol involving animals was approved by the Animal Ethics Committee of the No. 215 Hospital of Shaanxi Nuclear Industry.

\section{Patient consent for publication}

Not applicable.

\section{Competing interests}

The authors declare that they have no competing interests.

\section{References}

1. Ferlay J, Soerjomataram I, Dikshit R, Eser S, Mathers C, Rebelo M, Parkin DM, Forman D and Bray F: Cancer incidence and mortality worldwide: Sources, methods and major patterns in GLOBOCAN 2012. Int J Cancer 136: E359-E386, 2015.

2. Torre LA, Bray F, Siegel RL, Ferlay J, Lortet-Tieulent J and Jemal A: Global cancer statistics, 2012. CA Cancer J Clin 65: 87-108, 2015.

3. Guo X and Cui Z: Current diagnosis and treatment of pancreatic cancer in China. Pancreas 31: 13-22, 2005.

4. Long J, Luo GP, Xiao ZW, Liu ZQ, Guo M, Liu L, Liu C, Xu J, Gao YT, Zheng Y, et al: Cancer statistics: Current diagnosis and treatment of pancreatic cancer in Shanghai, China. Cancer Lett 346: 273-277, 2014

5. Bosetti C, Bertuccio P, Negri E, La Vecchia C, Zeegers MP and Boffetta P: Pancreatic cancer: overview of descriptive epidemiology. Mol Carcinog 51: 3-13, 2012.

6. Hidalgo M: Pancreatic cancer. N Engl J Med 362: 1605-1617, 2010.

7. Bartel DP: MicroRNAs: Genomics, biogenesis, mechanism, and function. Cell 116: 281-297, 2004.

8. Yang S, Zhang Y, Zhao X, Wang J and Shang J: microRNA-361 targets Wilms' tumor 1 to inhibit the growth, migration and invasion of non-small-cell lung cancer cells. Mol Med Rep 14: 5415-5421, 2016.

9. Lin P, Pang Q, Wang P, Lv X, Liu L and Li A: The targeted regulation of Gli1 by miR-361 to inhibit epithelia-mesenchymal transition and invasion of esophageal carcinoma cells. Cancer Biomark 21: 489-498, 2018.

10. Isaji S, Kawarada Y and Uemoto S: Classification of pancreatic cancer: Comparison of Japanese and UICC classifications. Pancreas 28: 231-234, 2004

11. Livak KJ and Schmittgen TD: Analysis of relative gene expression data using real-time quantitative PCR and the 2(-Delta Delta C(T)) method. Methods 25: 402-408, 2001.

12. Peng DY, Song H and Liu LB: Resveratrol-downregulated phosphorylated liver kinase B1 is involved in senescence of acute myeloid leukemia stem cells. J Huazhong Univ Sci Technolog Med Sci 35: 485-489, 2015.

13. Agarwal V, Bell GW, Nam JW and Bartel DP: Predicting effective microRNA target sites in mammalian mRNAs. Elife 4: $\mathrm{e} 05005,2015$.

14. Friedman RC, Farh KK, Burge CB and Bartel DP: Most mammalian mRNAs are conserved targets of microRNAs. Genome Res 19: 92-105, 2009.

15. Kang KA, Piao MJ, Madduma Hewage SR, Ryu YS, Oh MC, Kwon TK, Chae S and Hyun JW: Fisetin induces apoptosis and endoplasmic reticulum stress in human non-small cell lung cancer through inhibition of the MAPK signaling pathway. Tumour Biol 37: 9615-9624, 2016.

16. Mondal A, Biswas R, Rhee YH, Kim J and Ahn JC: Sulforaphene promotes $\mathrm{Bax} / \mathrm{Bcl} 2$, MAPK-dependent human gastric cancer AGS cells apoptosis and inhibits migration via EGFR, p-ERK1/2 down-regulation. Gen Physiol Biophys 35: 25-34, 2016.

17. Yeo TP, Hruban RH, Leach SD, Wilentz RE, Sohn TA, Kern SE, Iacobuzio-Donahue CA, Maitra A, Goggins M, Canto MI, et al: Pancreatic cancer. Curr Probl Cancer 26: 176-275, 2002.

18. Yonemori K, Kurahara H, Maemura $\mathrm{K}$ and Natsugoe $\mathrm{S}$ : MicroRNA in pancreatic cancer. J Hum Genet 62: 33-40, 2017.

19. Furtek SL, Backos DS, Matheson CJ and Reigan P: Strategies and approaches of targeting STAT3 for cancer treatment. ACS Chem Biol 11: 308-318, 2016.

20. Kıvanç G, Kalliope ND, Ezgi KA, Katrin JC, Jiaoyu A, Marina L and Hana A: The role of autophagy in pancreatic cancer: From bench to the dark bedside. Cell 9: 1063, 2020.

21. Xu Z, Li C, Qu H, Li H, Gu Q and Xu J: MicroRNA-195 inhibits the proliferation and invasion of pancreatic cancer cells by targeting the fatty acid synthase/Wnt signaling pathway. Tumour Biol 39: 1010428317711324, 2017. 
22. Zhang G, Liu D, Long G, Shi L, Qiu H, Hu G, Hu G and Liu S: Downregulation of microRNA-181d had suppressive effect on pancreatic cancer development through inverse regulation of KNAIN2. Tumour Biol 39: 1010428317698364, 2017.

23. Gu DN, Jiang MJ, Mei Z, Dai JJ, Dai CY, Fang C, Huang Q and Tian L: microRNA-7 impairs autophagy-derived pools of glucose to suppress pancreatic cancer progression. Cancer Lett 400: 69-78, 2017.

24. Kanitz A, Imig J, Dziunycz PJ, Primorac A, Galgano A, Hofbauer GF, Gerber AP and Detmar M: The expression levels of microRNA-361-5p and its target VEGFA are inversely correlated in human cutaneous squamous cell carcinoma. PLoS One 7: e49568, 2012.

25. Sun JJ, Chen GY and Xie ZT: MicroRNA-361-5p inhibits cancer cell growth by targeting CXCR6 in hepatocellular carcinoma. Cell Physiol Biochem 38: 777-785, 2016.

26. Ma F, Song H, Guo B, Zhang Y, Zheng Y, Lin C, Wu Y, Guan G, Sha $\mathrm{R}$ and Zhou Q: miR-361-5p inhibits colorectal and gastric cancer growth and metastasis by targeting staphylococcal nuclease domain containing-1. Oncotarget 6: 17404, 2015.

27. Liu D, Tao T, Xu B, Chen S, Liu C, Zhang L, Lu K, Huang Y, Jiang L, Zhang X, et al: MiR-361-5p acts as a tumor suppressor in prostate cancer by targeting signal transducer and activator of transcription-6 (STAT6). Biochem Biophys Res Commun 445: 151-156, 2014.

28. Chen W, Wang J, Liu S, Wang S, Cheng Y, Zhou W, Duan C and Zhang C: MicroRNA-361-3p suppresses tumor cell proliferation and metastasis by directly targeting SH2B1 in NSCLC. J Exp Clin Cancer Res 35: 76, 2016.

29. Liu J, Zhu J, Xiao Z, Wang X and Luo J: BBOX1-AS1 contributes to colorectal cancer progression by sponging hsa-miR-361-3p and targeting SH2B1. FEBS Open Bio: Jan 27, 2020 (Epub ahead of print).

30. Hu J, Li L, Chen H, Zhang G, Liu H, Kong R, Chen H, Wang Y, Li Y, Tian F, et al: MiR-361-3p regulates ERK1/2-induced EMT via DUSP2 mRNA degradation in pancreatic ductal adenocarcinoma. Cell Death Dis 9: 807, 2018.
31. Zhao D and Cui Z: MicroRNA-361-3p regulates retinoblastoma cell proliferation and stemness by targeting hedgehog signaling. Exp Ther Med 17: 1154-1162, 2019.

32. Lago TS, Silva JA, Lago EL, Carvalho EM, Zanette DL and Castellucci LC: The miRNA 361-3p, a regulator of GZMB and TNF is associated with therapeutic failure and longer time healing of cutaneous Leishmaniasis Caused by L. (viannia) braziliensis. Front Immunol 9: 2621, 2018.

33. Ji Y, Wang D, Zhang B and Lu H: MiR-361-3p inhibits $\beta$-amyloid accumulation and attenuates cognitive deficits through targeting BACE1 in Alzheimer's disease. J Integr Neurosci 18: 285-291, 2019.

34. Sun Y, Liu WZ, Liu T, Feng X, Yang N and Zhou HF: Signaling pathway of MAPK/ERK in cell proliferation, differentiation, migration, senescence and apoptosis. J Recept Signal Transduct Res 35: 600-604, 2015.

35. Li Y, Xi Z, Chen X, Cai S, Liang C, Wang Z, Li Y, Tan H, Lao $\mathrm{Y}$ and $\mathrm{Xu} \mathrm{H}$ : Natural compound Oblongifolin $\mathrm{C}$ confers gemcitabine resistance in pancreatic cancer by downregulating Src/MAPK/ERK pathways. Cell Death Dis 9: 538, 2018.

36. Roy SK, Srivastava RK and Shankar S: Inhibition of PI3K/AKT and MAPK/ERK pathways causes activation of FOXO transcription factor, leading to cell cycle arrest and apoptosis in pancreatic cancer. J Mol Signal 5: 10, 2010.

37. Yang K, Li Y, Lian G, Lin H, Shang C, Zeng L, Chen S, Li J, Huang C, Huang $\mathrm{K}$ and Chen Y: KRAS promotes tumor metastasis and chemoresistance by repressing RKIP via the MAPK-ERK pathway in pancreatic cancer. Int J Cancer 142: 2323-2334, 2018

(i) $($ This work is licensed under a Creative Commons Attribution-NonCommercial-NoDerivatives 4.0 International (CC BY-NC-ND 4.0) License. 